\title{
La transmisión de los valores y prácticas organizacionales cooperativas en las filiales extranjeras: El caso de la cooperativa multinacional Fagor Ederlan
}

\author{
Ignacio Bretos ${ }^{1}$ y Anjel Errasti ${ }^{2}$
}

Recibido: 31 de mayo de 2017 / Aceptado: 11 de septiembre de 2017

Resumen. En la economía globalizada actual, muchas cooperativas se están viendo obligadas a crecer internacionalmente mediante estrategias de inversión directa extranjera para mantener su competitividad y sobrevivir en los mercados. Tomando como referencia un estudio cualitativo sobre Fagor Ederlan, una de las mayores cooperativas multinacionales del Grupo Mondragón, este artículo examina las contradicciones planteadas por la internacionalización en las cooperativas, así como las tensiones y oportunidades para que dichas organizaciones reproduzcan las prácticas idiosincráticas del modelo cooperativo en las filiales extranjeras. El estudio de caso se basa en el análisis de diversa documentación interna cedida por la compañía y en 23 entrevistas en profundidad llevadas a cabo en las plantas domésticas e internacionales con personal de distintas áreas organizacionales y rangos jerárquicos. Los resultados sugieren que la internacionalización puede desencadenar fuertes tendencias degenerativas en las cooperativas, afectando fundamentalmente al nivel de participación de los trabajadores en la empresa. Por otro lado, evidencian la influencia de barreras institucionales, así como de factores derivados del rol estratégico de las filiales y de las relaciones de poder entre matriz y filial, que dificultan la transformación de las filiales extranjeras en cooperativas. El artículo también discute algunos aspectos que resultan esenciales para avanzar en la implementación de políticas que acerquen el modelo de gestión en las filiales extranjeras a los principios y valores cooperativos.

Palabras clave: Cooperativas; Internacionalización; Empresas multinacionales; Participación de los trabajadores; Relaciones laborales; Mondragón.

Claves Econlit: A130; F23; J54; M54.

\section{[en] Dissemination of the cooperative values and organizational practices in foreign subsidiaries: The case of the multinational co-op Fagor Ederlan}

\begin{abstract}
In today's global economy, cooperatives are being forced to grow internationally through foreign direct investment strategies so as to remain competitive and survive in markets. Drawing on a qualitative study of Fagor Ederlan, one of the largest multinational cooperatives of the Mondragon Group, this article examines the contradictions posed by internationalization in cooperatives, as well as the tensions and opportunities they face to replicate the cooperative model's idiosyncratic practices in foreign subsidiaries. The case study is based on the analysis of several internal documents provided
\end{abstract}

1 Universidad de Zaragoza, España

Dirección de correo electrónico: ibretos@unizar.es.

2 Universidad del País Vasco, España

Dirección de correo electrónico: a.errasti@ehu.es. 
by the company and 23 in-depth interviews carried out in the domestic and international plants with staff from different organizational areas and hierarchical ranks. The findings suggest that internationalization can trigger degenerative tendencies in cooperatives, which primarily affect the participation of workers in the company. Moreover, they reveal the influence of institutional barriers, as well as of factors related to the strategic role of subsidiaries and power relationships between headquarters and subsidiaries, that hamper the transformation of foreign subsidiaries into cooperatives. The article also discusses some essential aspects to make progress in the implementation of policies that bring the management model in such subsidiaries closer to the cooperative principles and values.

Keywords: Cooperatives; Internationalization; Multinational enterprises; Employee voice; Industrial relations; Mondragon.

Sumario. 1. Introducción. 2. Marco teórico y revisión de la literatura. 3. Metodología y datos. 4. Resultados empíricos del estudio de caso. 5. Discusión y conclusiones. 6. Referencias bibliográficas.

Cómo citar: Bretos, I. y Errasti, A. (2018) La transmisión de los valores y prácticas organizacionales cooperativas en las filiales extranjeras: El caso de la cooperativa multinacional Fagor Ederlan. REVESCO. Revista de Estudios Cooperativos, Primer Cuatrimestre, No 127, pp. 45-69. DOI: 10.5209/REVE.58395.

\section{Introducción}

En las dos últimas décadas marcadas por la intensificación del proceso de globalización, muchas cooperativas se han visto obligadas a crecer internacionalmente para sobrevivir en los mercados (Novkovic y Sena, 2007; Flecha y Ngai, 2014; Siebel, 2016). Sin embargo, algunas de estas cooperativas están expandiéndose mediante el establecimiento de filiales capitalistas en el extranjero (McMurtry y Reed, 2009), sufriendo así un proceso degenerativo basado en la adopción de estructuras capitalistas y la creación de empleo no cooperativo (Bretos y Marcuello, 2017). Como señalan Flecha y Ngai (2014), las cooperativas presentes en sectores altamente competitivos y globalizados se encuentran en una encrucijada. Por un lado, se están viendo forzadas a implantarse en el extranjero para mantener su competitividad. Por otro lado, también se espera que mantengan sus prácticas y valores cooperativistas y los transfieran a sus filiales internacionales de forma que puedan responder a las necesidades económicas, sociales y democráticas de los países donde están implantadas.

Este escenario plantea una pregunta de investigación fundamental: ¿Es posible reproducir el modelo cooperativo en las filiales extranjeras y transferir sus políticas y prácticas características centradas en la participación de los trabajadores? Aunque esta pregunta no es nueva en la literatura sobre las cooperativas, apenas ha sido contrastada empíricamente. De hecho, estudios recientes han solicitado investigar las contradicciones que genera la internacionalización en las cooperativas y las posibilidades para que extiendan el modelo cooperativo a sus filiales extranjeras (Bretos y Marcuello, 2017; Arzadun, 2015; Heras, 2014; Cheney et al., 2014; Flecha y Ngai, 2014; Radrigán y Barría, 2007). Por ejemplo, Cheney et al. (2014: 597-598) destacan que 'una cuestión clave para la investigación y la práctica es si dichas filiales [capitalistas] pueden convertirse en cooperativas y cómo pueden hacerlo, reconociendo no sólo las diferencias nacionales y culturales, sino también las preferencias de los propios empleados.' 
En esta línea, algunos trabajos han examinado el proceso de internacionalización de las cooperativas, centrándose fundamentalmente en las contradicciones generadas por el establecimiento de filiales capitalistas (Clamp, 2000; Errasti et al., 2003; Bakaikoa et al., 2004; Errasti et al., 2017). No obstante, estos trabajos carecen de un análisis detallado sobre cómo la internacionalización ha transformado las prácticas y valores cooperativistas en las matrices cooperativas y sobre cómo las diferencias socio-laborales entre matriz y filial son legitimadas o desafiadas por los directivos y trabajadores de ambos entornos. Por otro lado, algunos autores (Luzarraga, 2008; Luzarraga e Irizar, 2012; Bakaikoa et al., 2013; Flecha y Ngai, 2014) han examinado recientemente las iniciativas llevadas a cabo por algunas cooperativas para implantar sus prácticas y valores característicos en las filiales extranjeras. Estos trabajos concluyen que las cooperativas analizadas han sido capaces de reproducir el modelo cooperativo en sus filiales extranjeras, a pesar de que únicamente proporcionan evidencia de la implementación parcial de ciertas prácticas cooperativistas tales como la organización del trabajo en equipos auto-gestionados. Igualmente, dichos estudios tienen en cuenta exclusivamente la visión de la dirección de las matrices cooperativas, excluyéndose generalmente las perspectivas de los trabajadores y directivos de las plantas extranjeras.

Por tanto, aunque esta literatura previa ha proporcionado contribuciones esenciales en la comprensión de las cooperativas multinacionales, consideramos necesario aportar un estudio más detallado y crítico sobre las contradicciones generadas por la internacionalización y las tensiones que rodean la transformación de las filiales capitalistas en cooperativas. El Grupo Mondragón es particularmente adecuado para estudiar estas cuestiones. Nacido hace más de medio siglo en el País Vasco como una experiencia local, hoy en día, unas 25 cooperativas pertenecientes a la división industrial son empresas multinacionales que controlan 128 filiales en el extranjero y emplean a cerca de 13.000 personas en el exterior (Mondragón, 2016).

En concreto, este artículo aborda el caso de Fagor Ederlan, una de las mayores cooperativas multinacionales de Mondragón. La investigación se ha basado en diversa documentación interna proporcionada por la compañía y en 23 entrevistas realizadas en las plantas domésticas y filiales extranjeras de la cooperativa con personal de diversas áreas organizacional y rangos jerárquicos. Los resultados ponen en relieve, en primer lugar, los desafíos que plantea la internacionalización para que las cooperativas mantengan sus prácticas y valores cooperativos. Por otro lado, evidencian la influencia de barreras institucionales, así como de factores derivados del rol estratégico de las filiales y de las relaciones de poder entre matriz y filial, que dificultan la implementación del modelo cooperativo en las filiales extranjeras.

Tras esta introducción, el siguiente apartado revisa la literatura sobre la internacionalización de las cooperativas de Mondragón y la gestión en sus filiales extranjeras. El tercer apartado detalla la metodología y datos empleados en el estudio cualitativo. El cuarto apartado recoge los principales resultados de la investigación sobre Fagor Ederlan. Finalmente, se destacan las principales conclusiones del trabajo. 


\section{Marco teórico y revisión de la literatura}

Un tema de creciente interés en el ámbito del cooperativismo y la democracia organizacional se ha centrado en las tensiones existentes para que las cooperativas promuevan sus prácticas y valores originales mientras mantienen su competitividad y eficiencia económica bajo las nuevas presiones impuestas por la globalización de los mercados (Bretos y Marcuello, 2017; Cheney et al., 2014; Parker et al., 2014). Diversos trabajos sugieren que las cooperativas están sujetas a fuertes tensiones degenerativas derivadas de su necesario desarrollo en un entorno capitalista, que se traducen en última instancia en la dificultad para poner en práctica valores cooperativos tales como la solidaridad, la autogestión y la equidad (Atzeni y Ghigliani, 2007; Paranque y Willmott, 2014).

La literatura ha revelado crecientes tensiones en cooperativas que operan en mercados competitivos y globales, destacándose entre otros factores la tendencia hacia un mayor "gerencialismo" (Heras, 2014), la prevalencia de dinámicas oligárquicas en detrimento de la participación de los trabajadores (Varman y Chakrabarti, 2004), el nombramiento de directivos comprometidos en mayor medida con la eficiencia económica que con la cultura y valores cooperativos (Spear, 2004), o la reformulación y distorsión de los valores y prácticas cooperativistas en favor de las prerrogativas gerenciales relacionadas con el crecimiento y la competitividad internacional (Cathcart, 2013).

Sorprendentemente, a pesar de que el análisis de las cooperativas multinacionales es fundamental para avanzar en la comprensión de las tensiones y contradicciones que enfrentan las cooperativas en el contexto de la globalización actual (Cheney et al., 2014), la literatura previa apenas se ha centrado de manera explícita en este tipo de organizaciones. Como afirman Bretos y Marcuello (2017) en una reciente revisión de la literatura, existen dos cuestiones relacionadas con el desarrollo de las cooperativas en la globalización que requieren de un análisis más detallado y crítico: por un lado, las tendencias degenerativas que puede desencadenar la expansión internacional en las cooperativas y, por otro lado, las tensiones y oportunidades existentes en las cooperativas multinacionales para que promuevan las prácticas cooperativistas a lo largo de la red multinacional.

El Grupo Mondragón representa una de las pocas excepciones en que la literatura ha abordado en cierta medida estas cuestiones. No obstante, como sostienen recientes trabajos (Heras, 2014; Azkarraga et al., 2012), la experiencia cooperativa de Mondragón se ha mitificado en gran medida y, por tanto, suele estudiarse desde un punto de vista idealizado, sin valorar críticamente las tensiones y contradicciones reales. Ello se refleja claramente en cómo la literatura ha abordado la naturaleza y praxis de esta experiencia en el contexto de la globalización. Por ejemplo, Luzarraga e Irizar (2012: 114) caracterizan Mondragón como 'un caso de innovación cooperativa hacia una globalización centrada en la persona'. Forcadell (2005) destaca Mondragón como un ejemplo único en la combinación del éxito empresarial global con la utilización de métodos democráticos. Igualmente, MacLeod y Reed (2009) señalan que Mondragón es el modelo global más cercano a una verdadera empresa social.

En una línea similar, la literatura suele destacar que el modelo de internacionalización de Mondragón se ha basado en la 'multilocalización' 
(Luzarraga e Irizar, 2012), una estrategia expansionista y creativa ya que la implantación de nuevos negocios en el extranjero no implica el cierre de ninguna actividad preexistente (MacLeod y Reed, 2009). Se sostiene que, a diferencia del modelo de deslocalización adoptado por muchas multinacionales capitalistas, las cooperativas de Mondragón se han expandido internacionalmente sin que ello haya implicado el cierre de plantas productivas domésticas y la destrucción de empleo local (Luzarraga, 2008). De hecho, diversos trabajos apuntan que las cooperativas multinacionales del grupo Mondragón han sido capaces de generar más empleo que las cooperativas locales (las que no disponen de filiales en el extranjero) no sólo a nivel internacional, sino también en el País Vasco (Luzarraga e Irizar, 2012; Luzarraga et al., 2007). Por otro lado, la literatura reciente sugiere que la internacionalización ha dotado de flexibilidad a las cooperativas de Mondragón para resistir la crisis económica en mejores condiciones (Elortza et al., 2012).

No obstante, algunos trabajos han proporcionado un análisis más crítico de la internacionalización de Mondragón. Por ejemplo, recientes estudios que han analizado la situación de crisis y quiebra de Fagor Electrodomésticos (Errasti, 2013; Errasti, Bretos y Etxezarreta, 2016; Errasti, Bretos y Nunez, 2017) sugieren que el patrón característico de la multilocalización de las cooperativas de Mondragón parece no haberse cumplido en el caso de Fagor, buque insignia del grupo. En este sentido, señalan que a pesar de que la estrategia de multilocalización proveyó durante años de extraordinarios resultados a esta cooperativa, en sus últimos años de vida se destruyó empleo tanto en el exterior como en el País Vasco, pasando de más de 11.000 trabajadores antes de la crisis económica a tan sólo 5.500 trabajadores (alrededor de 2.000 en el País Vasco) un año antes de su quiebra.

Así mismo, algunos trabajos han analizado en cierta medida las contradicciones generadas por la internacionalización. Errasti et al. (2003) señalan que las cooperativas de Mondragón han desarrollado procesos de internacionalización en búsqueda de nuevos mercados emergentes o siguiendo a sus clientes para proveerles en los mercados internacionales donde se establecen. No obstante, los autores destacan que la problemática reside en el hecho de que estas filiales se han constituido como empresas capitalistas. En esta línea, Clamp (2000) señala que todas las filiales extranjeras son sociedades anónimas cuyos trabajadores son asalariados, produciéndose por tanto cierta ruptura con el modelo comunitario característico de las cooperativas de Mondragón.

Bakaikoa et al. (2004) ponen en relieve que los trabajadores de las filiales extranjeras no disponen de los mismos derechos que los socios cooperativistas de las matrices vascas, ya que el nivel de participación de dichos trabajadores en la gestión, el reparto de beneficios y la propiedad de sus empresas es muy reducido. Esta realidad se constata igualmente en otros trabajos que realizan un análisis más detallado de las relaciones laborales en las filiales extranjeras de Mondragón, concluyendo que en dichas filiales no hay rastro del modelo cooperativo de las matrices vascas y que las condiciones laborales son similares a las de otras multinacionales capitalistas del entorno (Errasti, 2015; Errasti et al., 2016; Errasti et al., 2017). Sin embargo, estos trabajos no arrojan demasiada luz sobre por qué las matrices cooperativas de Mondragón no han reproducido el modelo cooperativo 
en dichas filiales ni han transferido sus políticas características centradas en la participación de los trabajadores.

Al calor de las actuales estrategias de 'regeneración' que están desarrollando determinadas cooperativas de Mondragón (Bretos y Errasti, 2017; Bakaikoa et al., 2013), algunos trabajos han comenzado a analizar iniciativas de transformación de las filiales capitalistas. Bretos y Errasti $(2016,2017)$ examinan en detalle el proceso de transformación de una filial capitalista doméstica en cooperativa. Sin embargo, como reconocen en su estudio, es necesaria una mayor investigación sobre este tipo de experiencias en el ámbito extranjero, ya que la realidad en dichas filiales es completamente diferente y mucho más compleja que en las filiales domésticas.

En este sentido, muy pocos trabajos han analizado las iniciativas desarrolladas en Mondragón para acercar el modelo cooperativo a las filiales extranjeras. Luzarraga (2008) y Luzarraga e Irizar (2012) destacan algunas de las mejores prácticas de gestión de recursos humanos que han sido implantadas en el extranjero, tales como la organización del trabajo en equipos auto-gestionados, la introducción de programas de formación técnica y el intercambio de información con los empleados. En una línea similar, Flecha y Ngai (2014) concluyen que las cooperativas de Mondragón han sido capaces de mantener sus valores y prácticas cooperativistas durante la expansión internacional y de replicar el modelo de gestión cooperativo en las filiales extranjeras.

No obstante, se pueden destacar tres limitaciones fundamentales en estos trabajos. En primer lugar, el argumento de que las cooperativas de Mondragón han reproducido el modelo cooperativo en el exterior se basa simplemente en la aplicación parcial de algunas prácticas relacionadas con el modelo cooperativo en las filiales extranjeras. En segundo lugar, estos trabajos no incluyen generalmente las perspectivas y opiniones de los empleados y directivos locales de las filiales extranjeras o, en el mejor de los casos, son tratados como actores pasivos. En tercer lugar, sólo se hace referencia a ciertas barreras institucionales que dificultan la difusión internacional del modelo cooperativo, tales como las dificultades legales para transformar las filiales en cooperativas, eludiendo por tanto otros aspectos críticos como las relaciones de poder entre matriz y filial o los intereses propios de distintos actores.

Consecuentemente, parece necesario llevar a cabo un análisis más profundo y crítico acerca del proceso de internacionalización de las cooperativas, así como de la reproducción del modelo cooperativo en las filiales extranjeras y la transferencia de las políticas y prácticas basadas en la participación de los trabajadores.

\section{Metodología y datos}

El objetivo de esta investigación es explorar la reproducción del modelo cooperativo en las filiales extranjeras de cooperativas multinacionales. Debido a la naturaleza compleja y multidimensional que conlleva dicho análisis, se optó por diseñar un estudio empírico cualitativo siguiendo la metodología del 'estudio de casos contemporáneos', dada su idoneidad para comprender fenómenos sociales contemporáneos especialmente complejos en su contexto real (Yin, 2013; 
Villarreal-Larrinaga, 2017). De hecho, esta metodología ha sido ampliamente utilizada para estudiar las relaciones laborales en las empresas multinacionales y la difusión de prácticas organizacionales entre matriz y filial, donde es necesario tener en cuenta los diversos contextos sociales de la matriz y filiales así como las perspectivas de diferentes actores organizacionales como los trabajadores, directivos, y representantes sindicales. (Welch et al., 2011). Además, la metodología del estudio de casos es especialmente útil para arrojar luz sobre fenómenos de estudio en los que nuestra comprensión previa es todavía limitada (Eisenhardt y Graebner, 2007), gracias a su potencial para descubrir y examinar patrones y relaciones novedosas en la investigación y generar así ideas teóricas innovadoras (Welch et al., 2011).

Un aspecto clave de esta metodología reside en la adecuada identificación y selección de la unidad de análisis, es decir, del propio caso (Villarreal-Larrinaga, 2017). En este sentido, tras escrutar las experiencias existentes en diversas cooperativas multinacionales pertenecientes al grupo Mondragón, seleccionamos la cooperativa Fagor Ederlan porque es una de las más activas tanto en términos de expansión internacional como de extensión del modelo cooperativo a sus filiales. Por tanto, nuestra investigación trata un único caso con 'unidades incrustadas' (Baxter y Jack, 2008), es decir, la cooperativa y sus filiales.

Una vez seleccionado el caso, se procedió a la recogida de datos. Derivado de la naturaleza de esta metodología, se utilizaron diferentes fuentes para obtener los datos (Yin, 2013). Por un lado, tuvimos acceso a diversa documentación interna proporcionada por Fagor Ederlan que data desde principios de los años 90 (planes estratégicos y de gestión, estatutos, reglamentos de régimen interno, memorias de sostenibilidad, etc.). Así mismo, consultamos material disponible en su página web, revistas y boletines internos de Mondragón, entrevistas publicadas en medios digitales y notas de prensa. Por otro lado, la investigación se basó principalmente en entrevistas diseñadas para obtener descripciones narrativas detalladas y consistentes por parte de los propios actores involucrados en la cooperativa y sus filiales. Por tanto, la investigación tiene un carácter interpretativo (Gephart, 2004). En este sentido, el enfoque interpretativo proporciona 'una visión profunda de las experiencias desde el punto de vista de quienes las viven, asumiéndose que la realidad es una construcción social y que el investigador se erige como vehículo por medio del cual se revela dicha realidad' (Andrade, 2009: 43). A pesar de las limitaciones que ello conlleva como resultado de las posibles percepciones subjetivas tanto del investigador como del entrevistado, es una estrategia con fuertes potencialidades en tanto que la comunicación con las personas directamente involucradas en las organizaciones, instituciones y comunidades que forman parte del contexto de la investigación permite no sólo describir la realidad social, sino proporcionar conocimiento científico acerca de cómo puede ser transformada (Flecha y Soler, 2014).

En concreto, se llevaron a cabo un total de 23 entrevistas presenciales en profundidad, con una duración media de 90 minutos, entre 2012 y 2015. Las entrevistas se realizaron con socios cooperativistas, trabajadores asalariados y temporales, directivos, miembros del Consejo Rector y representantes sindicales del Grupo Fagor Ederlan (véase la tabla 1 para una descripción detallada). Algunos entrevistados solicitaron que no apareciese su puesto de trabajo concreto en la 
investigación para salvaguardar su anonimato, por lo que son identificados con su posición jerárquica en la organización (trabajador de planta, alto directivo, mando intermedio, etc.). Del conjunto de entrevistas, 12 se llevaron a cabo en plantas de la matriz y de la filial doméstica Fagor Ederlan Tafalla, 7 en la filial china Fagor Ederlan Auto-Parts Kunshan y 4 en la filial eslovaca Fagor Ederlan Slovensko. A pesar de que no tuvimos la oportunidad de visitar Fagor Ederlan Brasileira, la filial brasileña fue discutida extensamente en las entrevistas realizadas, ya que es una de las filiales de Mondragón donde más se ha avanzado en la implantación del modelo cooperativo.

Tabla. 1. Entrevistas en el Grupo Fagor Ederlan

\begin{tabular}{|c|c|c|}
\hline Entrevistas & Posición & Compañia \\
\hline Entrevista 1 & Presidente del Grupo Fagor Ederlan & Fagor Ederlan S. Coop. \\
\hline Entrevista 2 & Director General de Recursos Humanos & Fagor Ederlan S. Coop. \\
\hline Entrevista 3 & Alto directivo (Consejo Rector) & Fagor Ederlan S. Coop. \\
\hline Entrevista 4 & Socio trabajador de planta & Fagor Ederlan S. Coop. \\
\hline Entrevista 5 & Socio trabajador de planta & Fagor Ederlan S. Coop. \\
\hline Entrevista 6 & Alto directivo (Consejo Rector) & Fagor Ederlan Tafalla \\
\hline Entrevista 7 & Jefe de personal & Fagor Ederlan Tafalla \\
\hline Entrevista 8 & Representante sindical & Fagor Ederlan Tafalla \\
\hline Entrevista 9 & Socio trabajador de planta & Fagor Ederlan Tafalla \\
\hline Entrevista 10 & Socio trabajador de planta & Fagor Ederlan Tafalla \\
\hline Entrevista 11 & Trabajador asalariado de planta & Fagor Ederlan Tafalla \\
\hline Entrevista 12 & Trabajador asalariado de planta & Fagor Ederlan Tafalla \\
\hline Entrevista 13 & Gerente de planta & $\begin{array}{l}\text { Fagor Ederlan Auto- } \\
\text { Parts Kunshan }\end{array}$ \\
\hline Entrevista 14 & Mando intermedio local & $\begin{array}{l}\text { Fagor Ederlan Auto- } \\
\text { Parts Kunshan }\end{array}$ \\
\hline
\end{tabular}




\begin{tabular}{|c|c|c|c|c|}
\hline Entrevista 15 & Mando intermedio local & & $\begin{array}{l}\text { Fagor Ederlar } \\
\text { Parts Kunshan }\end{array}$ & Auto- \\
\hline Entrevista 16 & Directivo expatriado & & $\begin{array}{l}\text { Fagor Ederlar } \\
\text { Parts Kunshan }\end{array}$ & Auto- \\
\hline Entrevista 17 & Directivo expatriado & & $\begin{array}{l}\text { Fagor Ederlan } \\
\text { Parts Kunshan }\end{array}$ & Auto- \\
\hline Entrevista 18 & Trabajador asalariado de planta & & $\begin{array}{l}\text { Fagor Ederlar } \\
\text { Parts Kunshan }\end{array}$ & Auto- \\
\hline Entrevista 19 & Trabajador asalariado de planta & & $\begin{array}{l}\text { Fagor Ederlar } \\
\text { Parts Kunshan }\end{array}$ & Auto- \\
\hline Entrevista 20 & Gerente de planta & & $\begin{array}{l}\text { Fagor } \\
\text { Slovensko }\end{array}$ & Ederlan \\
\hline Entrevista 21 & $\begin{array}{l}\text { Directivo expatriado de } \\
\text { Humanos }\end{array}$ & Recursos & $\begin{array}{l}\text { Fagor } \\
\text { Slovensko }\end{array}$ & Ederlan \\
\hline Entrevista 22 & Representante sindical & & $\begin{array}{l}\text { Fagor } \\
\text { Slovensko }\end{array}$ & Ederlan \\
\hline Entrevista 23 & Trabajador asalariado de planta & & $\begin{array}{l}\text { Fagor } \\
\text { Slovensko }\end{array}$ & Ederlan \\
\hline
\end{tabular}

Fuente: elaboración propia

Por medio de cuestionarios semiestructurados, las entrevistas contrastaron fundamentalmente las perspectivas de tres tipos de actores organizacionales clave: la dirección, los trabajadores y sus representantes. En concreto, las entrevistas con los miembros de las plantas domésticas abordaron los desafíos que presenta la internacionalización para el mantenimiento de las prácticas cooperativas, así como las tensiones que percibían en la transformación cooperativa de las filiales extranjeras. En el caso de los trabajadores también se prestó especial atención a la visión sobre su participación en la cooperativa. En las filiales extranjeras, las entrevistas se centraron principalmente en la autonomía de dichas filiales y en la difusión de prácticas organizacionales desde la matriz. Las entrevistas con los trabajadores y representantes sindicales también trataron aspectos relacionados con las relaciones laborales y las condiciones de trabajo en las plantas extranjeras. Así mismo, también se plantearon preguntas a los miembros locales de las plantas extranjeras sobre su identidad en la empresa y su percepción del modelo cooperativo. Las entrevistas con los altos cargos se llevaron a cabo en las sedes de las empresas, visitándose además los talleres y otros lugares de trabajo. En cambio, buena parte de las entrevistas con las personas que no ostentaban puestos de dirección se realizaron fuera del lugar de trabajo, en diferentes espacios como cafeterías, universidades o asociaciones, evitando así el 'silencio organizacional' y favoreciendo la obtención de información crítica y veraz (Morrison y Milliken, 2000).

Las entrevistas se llevaron a cabo principalmente en castellano. No obstante, algunas entrevistas en China fueron traducidas simultáneamente al inglés por un 
intérprete. La inmensa mayoría de entrevistas fueron grabadas, aunque en otras ocasiones los propios entrevistados declinaron esta opción por motivos de privacidad. En estos casos, se tomaron notas manuscritas detalladas durante las entrevistas. Las entrevistas fueron transcritas y enviadas posteriormente a los entrevistados correspondientes para subsanar posibles errores y obtener su visto bueno. Tras esta etapa, se procedió al análisis de los datos de las entrevistas y fuentes secundarias.

Cabe destacar que, con el objetivo de aumentar el rigor y validez de los resultados del estudio de caso, se emplearon varias técnicas para la triangulación de los datos. Por un lado, se siguió una 'triangulación metodológica', derivada del uso de diferentes técnicas en la recogida de datos (Stake, 2005). En este sentido, las narrativas obtenidas de las entrevistas se contrastaron, cuando fue posible, con la información contenida en otras fuentes secundarias como, por ejemplo, los informes anuales y memorias de sostenibilidad de la compañía y las noticias de prensa. Por otro lado, también se procedió a la 'triangulación de encuestados' (Stake, 2005). En esta línea, un procedimiento clave consistió en contrastar la información proporcionada por entrevistados de distintos rangos jerárquicos de la cooperativa a quienes se realizaron las mismas preguntas. Por ejemplo, la información proporcionada por los directivos de la cooperativa acerca de la participación de los trabajadores fue contrastada con las experiencias compartidas por los propios trabajadores. Esta cuestión es especialmente relevante en el ámbito de Mondragón ya que, como señala Heras (2014), el discurso corporativo empleado por los directivos suele estar desvinculado de las prácticas reales que tienen lugar en el día a día de las cooperativas. Otro procedimiento clave consistió en contrastar las narrativas de los entrevistados pertenecientes a distintas unidades de la empresa multinacional. Por ejemplo, se contrastaron las afirmaciones realizadas por directivos de la matriz acerca de la gestión de recursos humanos y condiciones laborales en las filiales extranjeras con las narrativas de los propios miembros locales de dichas filiales. Como destacan Welch et al. (2011), este aspecto es fundamental para estudiar las empresas multinacionales debido al carácter 'multi-locacional' de estas organizaciones.

El análisis de los materiales se llevó a cabo siguiendo, en la línea de lo propuesto por Aguzzoli y Geary (2014: 596), una 'forma cualitativa de análisis de contenido' en la cual 'la unidad básica de análisis fue la frase relacionada con palabras clave agrupadas como categorías'. El 'análisis cualitativo del contenido' es definido por Hsieh y Shannon (2005: 1278) como 'un método de investigación para la interpretación subjetiva del contenido de los datos de texto a través de un proceso de clasificación sistemática de codificación e identificación de temas o patrones'. Dado el escaso conocimiento previo sobre el fenómeno de nuestra investigación, adoptamos un 'enfoque convencional' en el análisis cualitativo del contenido (Hsieh y Shannon, 2005), el cual implica básicamente que las categorías se desarrollan inductivamente. Es decir, en lugar de aplicar códigos y patrones derivados de la teoría, se permitió que las categorías emergieran directamente de los datos obtenidos.

En concreto, el análisis de contenido comenzó con un proceso de codificación abierta, en el que se asignaron palabras clave a extractos de texto que, posteriormente, fueron agrupados en temas emergentes. En este sentido, se 
identificaron temas clave como, por ejemplo, "internacionalización", "participación", "transferencia internacional de prácticas", o "autonomía de las filiales". A medida que avanzó el análisis del contenido, las palabras clave y los temas evolucionaron y se refinaron, generando así categorías más precisas a través de un proceso iterativo en el que surgieron nuevas subcategorías y otras se fusionaron, dividieron y eliminaron (Welch et al., 2011). Finalmente, tras un proceso de 'abstracción' por medio del cual las categorías se agrupan bajo encabezamientos de orden superior (Hsieh y Shannon, 2005), se identificaron dos categorías principales a partir de las narrativas de los entrevistados: las ventajas y tensiones derivadas de la internacionalización en Fagor Ederlan; y la transferencia del modelo cooperativo y de las políticas de participación a las filiales extranjeras. Consecuentemente, los resultados de la investigación se presentan en base a dichas categorías.

\section{Resultados empíricos del estudio de caso}

Fagor Ederlan, fundada en 1963 como resultado de la fusión de tres pequeñas empresas de fundición, es una de las cooperativas industriales pioneras que dieron lugar al actual Grupo Mondragón. Hoy en día es un proveedor global líder de automoción dedicado al diseño, desarrollo y fabricación de componentes metálicos de freno-suspensión y motor-transmisión. En 1990, Fagor Ederlan inició su expansión en el mercado doméstico con la adquisición de Victorio Luzuriaga S.A., y en 1998 comenzó su andadura internacional a través de un contrato con General Motors que supuso la apertura de una planta en Brasil.

Además de las 8 plantas de la matriz vasca, Fagor Ederlan dispone de las filiales domésticas Fagor Ederlan Tafalla, Fagor Ederlan Borja y Victorio Luzuriaga Usurbil; y de las filiales extranjeras Fagor Ederlan Brasileira (Brasil), Fagor Ederlan Slovensko (Eslovaquia) y Fagor Ederlan Auto-Parts Kunshan (China). Además, en 2015 firmó una alianza estratégica con el Grupo Industrial Saltillo para abrir una nueva planta en México. Por otro lado, Fagor Ederlan dispone del Centro Tecnológico Edertek en Mondragón y cuenta con 2 alianzas productivas en EEUU y Corea, y 8 oficinas comerciales en Europa, Asia y Norte América. En 2015, el Grupo contaba con una plantilla de 3.585 personas, las ventas ascendieron a 673 millones de euros (correspondiendo alrededor del $70 \%$ a ventas internacionales), y se realizaron inversiones valoradas conjuntamente en 43 millones de euros (Fagor Ederlan, 2016).

\subsection{El crecimiento internacional de Fagor Ederlan: ventajas y tensiones}

La historia del crecimiento de Fagor Ederlan se explica de manera paralela a la de otras cooperativas del Grupo Mondragón que, para mantener su competitividad y salvaguardar los empleos de los socios cooperativistas en las plantas vascas, han debido desarrollar una intensa estrategia de internacionalización desde los años 90 (Flecha y Ngai, 2014). Dadas las características del sector de automoción, Fagor Ederlan se ha expandido internacionalmente siguiendo a sus clientes. Como destacaba un directivo de Fagor Ederlan, 
Estamos en un sector altamente competitivo y globalizado como es la automoción. Si queremos mantener nuestra competitividad $\mathrm{y}$, en última instancia, generar empleo y desarrollo aquí y en el extranjero, debemos seguir a nuestros clientes y estar presentes en los principales mercados mundiales del sector. (Entrevistado 3)

Al igual que en el conjunto de Mondragón, la estrategia de expansión internacional se ha basado en el modelo de multilocalización. De esta forma, sólo se han transferido al extranjero actividades que ya no eran rentables en el País Vasco, sin que ello haya sido en detrimento del empleo local. De hecho, la estrategia internacional de multilocalización ha proporcionado extraordinarios resultados económicos a Fagor Ederlan, ha favorecido la creación de empleo tanto en el exterior como en el País Vasco y, en definitiva, ha fortalecido la posición de competitiva de la cooperativa en el mercado internacional. En este sentido, el empleo pasó en Fagor Ederlan de 1.312 trabajadores en 1999, apenas iniciada la internacionalización, a casi 3.600 empleos en 2015, aumentando además el empleo en las plantas vascas de la matriz de 1.452 a 1.641 trabajadores entre 2003 y 2008 (Fagor Ederlan, 2009, 2016). Como señalaba el mismo entrevistado,

Nuestro modelo de internacionalización se basa en la multilocalización. Esta estrategia ha sido clave para adaptarnos a las nuevas exigencias de la globalización. Nos ha permitido atender a las demandas globales de nuestros clientes mientras generábamos empleo y riqueza en el País Vasco y en el extranjero, incluso en estos últimos años de crisis. (Entrevistado 3)

Sin embargo, la estrategia de crecimiento internacional de Fagor Ederlan, al igual que en el resto de cooperativas multinacionales de Mondragón, se ha basado en el establecimiento de filiales capitalistas cuyo capital pertenece a la cooperativa matriz situada en el País Vasco. Como señalábamos, los trabajadores de dichas filiales no participan en el capital, el reparto de beneficios, la elección de los órganos de gobierno, ni en la gestión general de la empresa, como sí hacen los socios cooperativistas de la matriz (Bakaikoa et al., 2004).

Por tanto, Fagor Ederlan y otras cooperativas multinacionales de Mondragón se han transformado en híbridos 'coopitalistas' (Errasti, 2013, 2015), configuradas por un núcleo cooperativo (matriz) y una periferia capitalista (filiales). Esto implica una clara ruptura con los principios y valores cooperativistas. El modelo cooperativo se basa en la autogestión y el control directo de las actividades y los procesos por parte de los propios trabajadores involucrados en ellos. Sin embargo, esta solución 'coopitalista' implica que las decisiones sobre las filiales son tomadas en la matriz, desligadas por tanto de la involucración directa y la participación de los propios trabajadores.

Por otro lado, el incremento en la dimensión de la organización puede hacer disminuir el nivel de participación de los socios. Por un lado, porque pueden percibir que el impacto de su participación en la configuración de las políticas y objetivos organizacionales es mucho menor y, por otro lado, porque es más difícil que desarrollen una fuerte identificación y compromiso con la cooperativa. Estas tendencias parecen constatarse en cierta medida en Fagor Ederlan y otras cooperativas de Mondragón (véase también Errasti et al., 2017 para el caso de 
Fagor Electrodomésticos). Algunos socios sugirieron que el mayor tamaño organizacional derivado de la internacionalización ha influido en el grado y naturaleza de su participación en la matriz cooperativa. Tal y como reconocía uno de ellos,

El nivel de participación [de los socios] en las asambleas generales y otros espacios de decisión ha ido disminuyendo progresivamente en los últimos años [...] El mayor tamaño de la empresa y la complejidad en las decisiones que debemos tomar al estar presentes en un sector tan global y dinámico pueden haber sido razones fundamentales. (Entrevistado 5)

Igualmente, como resultado de este crecimiento, así como de las mayores presiones de la globalización para ser competitivas y eficientes económicamente, las cooperativas pueden verse obligadas a desarrollar nuevas estructuras de gestión y depositar un elevado poder de control en gerentes que, en muchas ocasiones, están más comprometidos con la eficiencia económica que con los valores y cultura cooperativa (Spear, 2004). Como destacaba el Director de Recursos Humanos de Fagor Ederlan,

Conforme la empresa ha ido creciendo en los últimos años y hemos debido atender a las demandas de nuestros clientes internacionales y cumplir con los rigurosos estándares del sector, hemos ido incorporando profesionales externos en la gestión y dirección con talento y experiencia, lo cual ha sido clave para estar donde estamos actualmente. (Entrevistado 2)

En definitiva, este escenario puede afectar al grado de participación democrática de los trabajadores en la gestión. La prevalencia de los objetivos de productividad y eficiencia económica puede llevar a que las cooperativas implanten una cultura débil de participación, centrada exclusivamente en el área de trabajo y articulada a través de programas de gestión convencionales como la Gestión de la Calidad Total, los cuales están orientados a promover la motivación de los trabajadores y su compromiso con los objetivos marcados por la gerencia de la empresa (Cheney, 2002; Heras, 2014). Estas dinámicas han sido evidentes en Mondragón. Por ejemplo, en 2003, Fagor Ederlan implantó el modelo de las denominadas 'minicompañías', que destaca como la concreción práctica de la gestión de la calidad para la organización del trabajo. Tal y como señalaba el mismo entrevistado citado anteriormente, el objetivo principal era incrementar la productividad a partir del compromiso de los trabajadores con los objetivos empresariales.

[Las mini-compañías] son formas organizativas diseñadas para las áreas productivas, con la idea de que cada una de ellas funcione como una pequeña empresa en busca de la satisfacción de los clientes internos [otras mini-compañías] y externos. Con ellas se quiere dar más autonomía a los trabajadores, siendo ellos quienes decidan y solucionen los problemas en el mismo ámbito en el que se generan con el soporte de todos los departamentos y áreas de la empresa [...] Su finalidad es incrementar la eficiencia y productividad mediante la implicación de las personas. (Entrevistado 2) 


\subsection{La transferencia del modelo cooperativo y de las políticas de participación de los trabajadores a las filiales extranjeras}

En los últimos años, algunas cooperativas multinacionales de Mondragón están tratando de impulsar iniciativas que tienen como objetivo transformar las filiales capitalistas en cooperativas o, al menos, fomentar en ellas la participación de los trabajadores en la propiedad, distribución de beneficios y gestión de la empresa (Flecha y Ngai, 2014). Fagor Ederlan se ha erigido como una de las cooperativas más activas en este ámbito. En el ámbito doméstico, ha transformado tres de sus plantas en cooperativas. Por un lado, la filial 'FIT Automoción Bergara', situada en el País Vasco, pasó a ser una planta productiva de la propia matriz y los trabajadores se convirtieron en socios de la cooperativa. Actualmente, la sociedad participada 'Victorio Luzuriaga Usurbil', también situada en el País Vasco, está inmersa en el mismo proceso. Por otro lado, en 2008, la antigua sociedad anónima 'Victorio Luzuriaga Tafalla', situada en Navarra, se transformó en la actual cooperativa mixta 'Fagor Ederlan Tafalla' (véase Bretos y Errasti, 2016, 2017 para un análisis detallado).

Sin embargo, en el ámbito extranjero es mucho más complejo desarrollar este tipo de iniciativas. En primer lugar, un factor clave es la mayor 'distancia institucional' (Kostova, 1999), es decir, las diferencias institucionales a nivel regulatorio, normativo y cognitivo entre el país de origen de la empresa multinacional y el país receptor donde está localizada la filial (Kostova y Roth, 2002). En este sentido, una de las principales limitaciones que impiden la implantación de filiales en el extranjero bajo la fórmula cooperativa puede derivar del hecho de que en el país receptor no exista una legislación cooperativa sólida o similar a la del País Vasco (Flecha y Ngai, 2014). Esta distancia institucional regulatoria, que hace referencia a las diferencias en las reglas y leyes entre los marcos institucionales de dos países (Scott, 1995; Kostova, 1999), fue evidente cuando Fagor Ederlan trató de transformar su filial brasileña en cooperativa. Esta iniciativa sobresale como el único intento en Mondragón de transformar una filial extranjera en cooperativa. Como destacaba el Presidente del Grupo Fagor Ederlan,

En Brasil analizamos las posibilidades de transformar una planta en cooperativa, pero las diferencias legales entre nuestras legislaciones sobre cooperativas impidieron seguir adelante con el proyecto. (Entrevistado 1)

A raíz de la experiencia brasileña, desde Fagor Ederlan se empezaron a plantear otras alternativas para implantar el modelo cooperativo en las filiales extranjeras, principalmente a través de la participación de los trabajadores en la propiedad, beneficios y gestión de sus empresas. Como señalaba el mismo entrevistado,

No creemos que lo importante sea que las filiales se transformen jurídicamente en cooperativas, sino fomentar en ellas la democracia y la participación de los trabajadores [...] El objetivo es extender en ellas la cultura cooperativa y al menos compartir modelos de gestión. (Entrevistado 1) 
En este contexto, destacan los avances alcanzados en la filial brasileña gracias a la menor distancia institucional tanto en su dimensión normativa, basada en los valores, creencias y normas sociales de los individuos de un país, como en su dimensión cognitiva, referida a las percepciones e interpretaciones de la realidad y el entorno ampliamente compartidas por los individuos de un país (Scott, 1995; Kostova y Roth, 2002). De esta forma, se ha logrado avanzar en la transferencia de determinadas políticas de recursos humanos y prácticas socio-laborales más próximas al modelo de gestión cooperativo de la matriz. En este sentido, la mayor proximidad con la cultura cooperativa brasileña ha sido un factor determinante. En Brasil existe una sólida tradición cooperativa y cultura de autogestión, a pesar de que durante mucho tiempo las empresas cooperativas han sido percibidas negativamente en el imaginario colectivo de la sociedad brasileña debido a la herencia de las llamadas 'pseudo-cooperativas'; situación que se ha ido revirtiendo en los últimos años (Sarria-Icaza, 2008). El Director de Recursos Humanos de Fagor Ederlan se expresaba en los siguientes términos,

En la filial brasileña hemos conseguido avanzar significativamente en la implantación del modelo cooperativo debido a que allí existe una cultura cooperativa más cercana a la nuestra, y a que es la planta que lleva más tiempo abierta en el extranjero, lo que nos ha permitido trabajar con ellos en estos aspectos a largo plazo. (Entrevistado 2)

En esta filial se ha implantado un balance social con indicadores de desempeño económico, social y medioambiental, que permiten comparar y equiparar las características de la filial con las de la matriz (Luzarraga e Irizar, 2012). Por otro lado, se han implantado las políticas de prevención de riesgos laborales y algunas medidas de equidad social similares a las de la matriz, como la estructura salarial equitativa dividida en 6 categorías. Estas medidas, junto al resto de condiciones laborales, son revisadas anualmente para mantenerlas en niveles similares o superiores a las del entorno de la filial. También se han integrado los programas de formación técnica para los trabajadores y las políticas de promoción interna, reduciendo de esta manera la rotación $\mathrm{y}$ fomentando la estabilidad laboral (Luzarraga, 2008).

En el caso de la filial eslovaca y especialmente la china es todavía más complejo avanzar en la implantación del modelo cooperativo debido a una mayor distancia institucional en sus tres dimensiones. Por un lado, en China no existe actualmente una legislación que ampare a las cooperativas de trabajo. Por otro lado, China tiene una herencia de estilos directivos autocráticos estructurados en poderes fuertemente centralizados, así como una cultura laboral y unas dinámicas del mercado laboral muy diferentes a las del País Vasco (Xiaowen, 2007). Todo ello dificulta la implantación del modelo cooperativo, basado en la toma de decisiones democrática y la participación de los trabajadores en la gestión. Como indicaba el gerente de la filial china,

No se ha puesto sobre la mesa la posibilidad de transformar esta filial en una cooperativa. Las dificultades legales serían inmensas y, además, sería muy 
complicado que los trabajadores se adaptasen al modo de funcionamiento de una cooperativa. (Entrevistado 13)

Así mismo, es destacable que los trabajadores de la filial china no eran conscientes de lo que significaba exactamente una cooperativa de trabajo. Una vez se les explicó brevemente, tampoco mostraron una clara disposición a adoptar este modelo. Uno de ellos lo expresó claramente de la siguiente manera:

No creo que estemos preparados aquí [en Kunshan] para trabajar en una cooperativa. Los trabajadores no estaríamos dispuestos a invertir nuestro dinero en la cooperativa, ya que lo necesitamos para salir adelante. Apenas tenemos las vacaciones y trabajamos muchas horas extra para ahorrar. Además, tengo mis dudas de si seríamos capaces de gestionar la empresa por nosotros mismos. No estamos acostumbrados a tomar decisiones en grupo. Simplemente hacemos lo que nos dicen nuestros supervisores. (Entrevistado 19)

Sin embargo, esta filial se sitúa en el Parque Industrial de Kunshan, uno de los clústeres internacionales donde se localizan filiales de diversas cooperativas de Mondragón. Dichos clústeres proporcionan a las cooperativas de Mondragón mayores oportunidades para implantar sus políticas socioeconómicas, por ejemplo, en el ámbito de la gestión de recursos humanos (Luzarraga e Irizar, 2012). Como indicaba el Director de Recursos Humanos,

No tengo duda de que la localización de la filial en Kunshan nos permitirá avanzar en los próximos años en un modelo de trabajo y gestión mucho más próximo al que tenemos en nuestras cooperativas. Ya existe un sólido modelo de inter-cooperación y generación de sinergias con otras filiales de Mondragón y empresas locales. (Entrevistado 2)

En una línea similar se expresaba un socio de Fagor Ederlan,

No creo que sea imposible implantar a largo plazo ciertos aspectos del modelo cooperativo en la filial china. La filial se abrió recientemente [en 2012] y todavía no está a pleno rendimiento, ya que se alcanzarán los 100 empleos en 2017 (...). Es una réplica de nuestra planta principal en Eskoriatza [País Vasco], por lo que se ha invertido mucho en la formación del personal. Algunos incluso han visitado nuestras instalaciones para aprender nuestro modelo de trabajo en equipo y para adquirir formación técnica y organizativa. (Entrevistado 4)

En cualquier caso, en líneas generales, los avances en la promoción de la participación de los trabajadores en la propiedad, beneficios y gestión de las filiales extranjeras han sido aparentemente limitados en Fagor Ederlan. En este sentido, los trabajadores de las filiales extranjeras no son propietarios ni participan en el reparto de beneficios. En términos de participación en la gestión no cabe duda de que, a través de las mini-compañías, los trabajadores tienen una amplia autonomía en la toma de decisiones relativas al área de trabajo, aunque no participan directamente en la toma de decisiones a nivel corporativo. 
En este contexto, existen también otros factores derivados del rol que juegan las filiales. Las cooperativas de Mondragón han adoptado un enfoque anglosajón en la gestión de sus filiales, caracterizado por un control centralizado y por la tendencia a transferir las prácticas y políticas de recursos humanos de forma estandarizada (Quintanilla et al., 2008). Así, las filiales extranjeras tienen un elevado grado de dependencia con respecto a las cooperativas matrices, con una autonomía muy limitada en los aspectos estratégicos, técnicos, financieros y comerciales; áreas que suelen estar gestionadas por expatriados o gerentes muy próximos a la matriz (Errasti, 2015; Errasti et al., 2016). La razón principal de la internacionalización en las cooperativas de Mondragón es mantener el empleo y la estabilidad laboral en sus matrices vascas y, para ello, retienen las funciones clave en términos de gestión, diseño de producto, inversión, $\mathrm{I}+\mathrm{D}$, etc. Como señalaba un directivo,

No podemos olvidar que nuestro principal objetivo es mantener y generar empleo en las plantas del País Vasco, al mismo tiempo que creamos empleo en el extranjero [...] Esto implica que tomaremos decisiones estratégicas teniendo esto en mente, y mantendremos nuestras actividades de mayor valor añadido en el País Vasco con el objetivo de que nuestras plantas sigan siendo rentables y podamos mantener el empleo de los socios [cooperativistas] aquí. (Entrevistado 3)

De esta forma, las cooperativas matrices de Mondragón sólo transfieren prácticas de gestión estandarizadas como los círculos de calidad y otras formas de organización del trabajo en pequeños grupos auto-gestionados. Estos sistemas, cuya finalidad principal es fomentar la productividad y flexibilidad, se erigen como modelos de participación superficial, centrados exclusivamente en el área de trabajo, y evaluados en términos del compromiso de los empleados con los objetivos marcados por la gerencia (Cheney, 2002). Este es el caso de Fagor Ederlan, que ha implantado el modelo de las 'mini-compañías' en las filiales extranjeras para fortalecer su desempeño económico y organizacional, y lograr así cierta consistencia interna entre las unidades multinacionales. Como destacaba un directivo expatriado en la filial china de Fagor Ederlan,

Nuestra tarea es consolidar la filial y hacer que sea rentable. Tratamos de facilitar la transferencia de las políticas y prácticas marcadas por la matriz bajo su supervisión [...]. Aquí, al igual que en el resto de las filiales, hemos implementado el mismo sistema de gestión de calidad total importado de la empresa matriz. (Entrevistado 17)

No obstante, parece que existen ciertas reticencias en la matriz no sólo para transformar directamente las filiales extranjeras en cooperativas, sino también para transferir políticas y prácticas que fomenten realmente la participación de los trabajadores en la propiedad, distribución de beneficios y gestión de dichas filiales. En este sentido, un factor fundamental es la percepción entre los socios trabajadores y directivos de la matriz de que los trabajadores de las filiales extranjeras no van a desarrollar una identidad y compromiso con los valores cooperativos y con la compañía tan sólido como el suyo. Esto parece provenir, al menos en parte, de la falta de relaciones, lazos y confianza entre los miembros de 
la matriz y los trabajadores de las filiales extranjeras, un aspecto habitual en las cooperativas multinacionales de Mondragón (Errasti, 2015; Errasti et al., 2016). En última instancia, dicha percepción genera dudas sobre el éxito de una hipotética transformación de una filial extranjera en cooperativa. Como declaró un socio trabajador de Fagor Ederlan,

No tenemos contacto con los trabajadores de las filiales extranjeras. Creo que es difícil que la transformación de una filial extranjera en cooperativa pueda tener éxito. Los valores cooperativos no se adquieren de la noche a la mañana. Además, no sé si los trabajadores de las filiales extranjeras desarrollarían una fuerte identidad con la empresa. Al final, si un proyecto de este tipo en una filial extranjera sale mal, esto nos afecta a todos. (Entrevistado 5)

De este modo, la transformación cooperativa de las filiales extranjeras es considerada como algo problemático en la matriz, ya que podría afectar a la estabilidad de todo el grupo empresarial y, por lo tanto, poner en peligro los puestos de trabajo de los socios trabajadores en las plantas vascas, generando así una clara contradicción con el objetivo de la internacionalización en Mondragón. Esto es consistente con el reciente estudio de Heras (2014) sobre Mondragón, que concluye que la estabilidad y seguridad laboral es el principal lazo que une a los socios con sus cooperativas, en detrimento de la identificación con la cultura y valores cooperativos. Un alto directivo de una filial doméstica de Fagor Ederlan lo puso de manifiesto en los siguientes términos:

Existe una estrecha relación entre los miembros de la matriz y nosotros. Sin embargo, la relación con los trabajadores, por ejemplo, de la filial china es inexistente (...). Creo que su cultura laboral está muy alejada de nuestros valores cooperativos y la forma de entender las relaciones de trabajo aquí (...). Si se transformase en cooperativa, no sé si su proyecto socio-empresarial tendría éxito. Además, ¿quién nos asegura que permanecerían en el grupo empresarial? Este escenario es arriesgado. Podría poner en peligro nuestros puestos de trabajo. (Entrevistado 6)

\section{Discusión y conclusiones}

Este artículo ha estado motivado por los recientes llamamientos en la literatura para examinar cómo las cooperativas multinacionales pueden replicar sus valores y prácticas organizacionales en las filiales extranjeras (entre otros, Bretos y Marcuello, 2017; Arzadun, 2015; Cheney et al., 2014). De esta forma, nuestros resultados también contribuyen a extender recientes investigaciones que han analizado la implantación del modelo cooperativo en las filiales domésticas de cooperativas multinacionales (Bretos y Errasti, 2016, 2017).

Los escasos estudios anteriores que han abordado nuestra pregunta de investigación sugieren que las cooperativas multinacionales de Mondragón han sido capaces de reproducir el modelo cooperativo en el exterior (Luzarraga, 2008; Luzarraga e Irizar, 2012; Bakaikoa et al., 2013; Flecha y Ngai, 2014). Estos 
trabajos han proporcionado contribuciones esenciales, señalando que dichas cooperativas han transferido a sus filiales extranjeras diversas prácticas asociadas al modelo cooperativo, tales como la implantación de equipos auto-gestionados y el fomento de la difusión de información y de la comunicación entre trabajadores y directivos. No obstante, a pesar de reconocer que ninguna de las filiales extranjeras de Mondragón ha sido transformada en cooperativa ni ha introducido de manera consistente las prácticas centrales asociadas al modelo cooperativo (es decir, la participación de los trabajadores en la propiedad, reparto de beneficios y gestión), estos estudios carecen de un análisis crítico que profundice en las razones subyacentes. Igualmente, es evidente que estos trabajos han pasado por alto el rol que juegan las relaciones de poder y los intereses en las cooperativas multinacionales y no han tenido en cuenta las perspectivas de actores clave como los trabajadores y directivos locales de las filiales extranjeras.

Como demuestra esta investigación, estos aspectos presentan un complejo escenario y resultan fundamentales para comprender los dilemas y paradojas que las cooperativas enfrentan para transformar genuinamente sus plantas extranjeras. En concreto, nuestros resultados muestran las posibilidades de implementar las prácticas características del modelo cooperativo en las filiales internacionales a pesar de que éstas no sean transformadas directamente en cooperativas, tal y como pone de manifiesto los avances alcanzados en la filial brasileña de Fagor Ederlan. No obstante, los resultados evidencian que la difusión de los valores y prácticas organizacionales cooperativas en las filiales extranjeras puede verse limitada, en primer lugar, por la influencia de dos barreras institucionales esenciales: las diferencias legislativas y las culturales. La primera hace referencia a la carencia de una legislación que ampare a las cooperativas de trabajo en el país receptor donde opera la filial, lo que dificulta significativamente su posible transformación en cooperativa. La segunda implica básicamente la falta de una tradición y cultura cooperativista en el entorno donde opera la filial, traduciéndose en la dificultad para que los trabajadores interpreten correctamente y legitimen las prácticas características del modelo cooperativo.

De esta forma, tal y como sugiere la teoría neo-institucional (Rosenzweig y Nohria, 1994; Kostova et al., 2008; Clark y Lengnick-Hall, 2012), las filiales muestran unas características propias sujetas al marco institucional de su entorno, por lo que las relaciones laborales en dichas filiales no dependen tanto de la naturaleza cooperativa de su matriz. Sin duda, la distancia institucional entre el país de origen de la empresa multinacional y el país receptor donde opera la filial juega un papel fundamental en este contexto (Kostova y Roth, 2002). Ello se evidencia realizando una comparativa entre la filial brasileña y la filial china de Fagor Ederlan. En Brasil se ha avanzado en mayor medida en la implantación de diversas prácticas cooperativistas debido a que existe una distancia cultural menos notable con el País Vasco, gracias especialmente a la presencia de una cultura cooperativa más cercana. Por ejemplo, la organización del trabajo se articula en torno a equipos de trabajo auto-gestionados (mini-compañías), se ha fomentado la difusión de información entre los trabajadores favoreciendo además su involucración en la toma de decisiones relativas al área de trabajo, y se ha avanzado en la introducción de políticas de equidad salarial. En cambio, las marcadas diferencias legales y culturales con China obstaculizan significativamente la introducción del modelo 
cooperativo en esta filial. Consecuentemente, la participación de los trabajadores en esta filial es significativamente más reducida, en línea con el entorno institucional en el que se encuentra localizada dicha filial (Xiaowen, 2007).

No obstante, en consonancia con el 'enfoque político' sobre la difusión de prácticas organizacionales en la empresa multinacional (por ejemplo, Dörrenbächer y Geppert, 2011; Ferner et al., 2012; Aguzzoli y Geary, 2014), nuestros resultados muestran que las relaciones de poder entre matriz y filial y los intereses propios de diferentes actores organizacionales en la empresa multinacional influyen en la difusión transnacional de prácticas de manera más significativa que las diferencias institucionales. Como hemos visto, las cooperativas de Mondragón se internacionalizan con el objetivo de mantener su competitividad y proteger los puestos de trabajo de los socios trabajadores en las plantas vascas. Para ello, ejercen un elevado control centralizado sobre las filiales, reteniendo los recursos estratégicos y procesos de alto valor añadido. De esta manera, pueden transferir políticas y prácticas de manera estandarizada a las filiales (Quintanilla et al., 2008), tales como el sistema de gestión de la calidad total a través de las mini-compañías, con el objetivo de fortalecer su eficiencia económica y lograr la consistencia interna a nivel global requerida en el conjunto del grupo empresarial para competir de manera eficaz en los mercados internacionales (Ferner et al., 2005). Sin embargo, es evidente que existen intereses propios entre los directivos y socios de la matriz que obstaculizan la implantación genuina y consistente del conjunto de prácticas que configuran el modelo cooperativo, ya que consideran que la mayor autonomía de las filiales extranjeras y la participación de sus trabajadores pueden ser perjudiciales para el control de la matriz cooperativa sobre el grupo y para la estabilidad laboral de los socios trabajadores.

Por otro lado, nuestra investigación aporta una comprensión más detallada sobre las contradicciones que plantea la internacionalización en las cooperativas. Hasta ahora, la literatura previa había puesto el énfasis en el dilema planteado por el establecimiento de filiales capitalistas, señalando sus incoherencias con la naturaleza y praxis cooperativa (Errasti et al., 2003; Bakaikoa et al., 2004; Errasti et al., 2016). En esta línea, el estudio de caso aquí presentado profundiza en dichas contradicciones e incoherencias $\mathrm{y}$, adicionalmente, permite extraer algunas conclusiones que van más allá de lo discutido en la literatura previa, evidenciando que la internacionalización puede intensificar tres dinámicas degenerativas clave en las cooperativas identificadas en el trabajo de Narvaiza et al. (2017): la degeneración constitucional, organizacional y de objetivos.

En primer lugar, los resultados muestran que las cooperativas multinacionales pueden sufrir un proceso de degeneración constitucional, basado en la adopción de estructuras y formas de organización capitalista, al desarrollar una estrategia de internacionalización sostenida en el modelo dual 'núcleo cooperativo-periferia capitalista', el cual implica una clara ruptura con los valores democráticos, las políticas centradas en el trabajador y los objetivos sociales característicos de estas organizaciones. En segundo lugar, las cooperativas multinacionales pueden estar sujetas a dinámicas de degeneración organizacional, en tanto que las presiones de la competitividad internacional y de la eficiencia económica, derivadas de operar en sectores globales, pueden llevar a las cooperativas a desarrollar una gestión profesionalizada que otorgue un excesivo poder de control a la gerencia en 
detrimento de la participación de los trabajadores. Esto puede observarse en cómo Fagor Ederlan ha implantado sistemas de gestión convencionales, como la Gestión de la Calidad Total, que han desplazado la auto-gestión hacia una cultura de participación débil, ya que este tipo de sistemas tienden a instaurar un modelo de participación centrado exclusivamente en el área de trabajo, controlado gerencialmente y evaluado en términos del compromiso de los trabajadores con los objetivos empresariales marcados desde arriba (Cheney, 2002; Heras, 2014). Por último, las cooperativas multinacionales pueden sufrir un proceso de degeneración de objetivos basado en la sustitución de los objetivos sociales cooperativistas por objetivos convencionales relativos a la búsqueda de beneficios y el crecimiento. Esta tendencia se ha podido ver intensificada en Fagor Ederlan por la prevalencia de una gestión tecnocrática y de un discurso gerencial más centrado en la eficiencia económica que en la cultura cooperativa.

En términos más amplios, estos resultados contribuyen también a los recientes debates sobre las tensiones que afrontan las cooperativas para poner en práctica sus valores característicos, tales como la democracia, la solidaridad o la autogestión, mientras son capaces de cumplir con los requerimientos de competitividad y eficiencia económica impuestos en el contexto de la globalización (Cathcart, 2013; Storey et al., 2014; Parker et al., 2014; Paranque y Willmott, 2014; Narvaiza et al., 2017).

Nuestro estudio también plantea algunas implicaciones más prácticas que permitan avanzar en la implantación del modelo cooperativo en las filiales extranjeras. En primer lugar, la generación de capital social a través de las relaciones entre matriz y filial es esencial, ya que fomenta la disposición a compartir información y conocimiento entre trabajadores de unidades distanciadas geográfica y culturalmente (Taylor, 2007). En la cooperativa matriz, puede generar una mayor determinación para transferir el modelo cooperativo y sus prácticas características centradas en la participación de los trabajadores. Igualmente, la confianza de la filial en la matriz puede facilitar enormemente la aceptación e implementación de las políticas y prácticas transferidas (Kostova y Roth, 2002). Así mismo, es fundamental que, previamente, las matrices promuevan programas de formación y educación sobre los principios y valores cooperativistas en las filiales extranjeras. De lo contrario, la transferencia de prácticas complejas, como la participación de los trabajadores en la gestión, podría fracasar debido al choque con las instituciones cognitivas del país receptor, ya que los trabajadores podrían tener dificultades para interpretar dichas prácticas correctamente (Ferner et al., 2005).

Por otro lado, las cooperativas deberían abandonar un enfoque 'etnocéntrico' (Perlmutter, 1969) basado en la idea de que el modelo cooperativo de su país de origen es también el más apropiado para las filiales extranjeras. Existen en el mundo diferentes enfoques culturales y legislaciones sobre las cooperativas de trabajo (Cheney et al., 2014). Por tanto, los proyectos de transformación de las filiales extranjeras deberían adaptarse a la cultura local y condiciones institucionales de sus entornos, integrando además las perspectivas de los trabajadores. Así mismo, resulta esencial que las cooperativas promuevan previamente la autonomía de las filiales, aproximándose así a modelos federativos que otorgan a las filiales un mayor poder de decisión y capacidad para desarrollar 
competencias independientes (Andersson y Holm, 2010). Consecuentemente, como sostiene la teoría sobre el desarrollo económico regional (Holm et al., 2003), estas filiales podrían ejercer una mayor influencia positiva en las economías locales. En definitiva, a través de la transformación de las filiales extranjeras, las cooperativas multinacionales podrían servir como palanca para promover mejoras sociales, económicas y democráticas en los territorios donde están presentes.

Finalmente, una importante limitación del estudio reside en el problema de la 'generalización' de nuestras conclusiones, debilidad común al desarrollar una investigación cualitativa basada en un único caso de estudio (Villarreal-Larrinaga, 2017). Nuestros resultados y conclusiones se refieren al caso de Fagor Ederlan y, por tanto, deben ser generalizados con cautela en el ámbito general de las cooperativas multinacionales. Por otro lado, a pesar de que se han contrastado las perspectivas de los diferentes actores organizacionales, el análisis de entrevistas y datos secundarios puede estar sujeto a las percepciones subjetivas del investigador (Eisenhardt y Graebner, 2007). Igualmente, este tipo de sesgos pueden provenir de los entrevistados ya que, como señala Heras (2014), los trabajadores pueden aprovechar las entrevistas para expresar sus frustraciones, ofreciendo así una visión distorsionada de la realidad. Por tanto, con el objetivo de contrastar y ampliar nuestros resultados en el ámbito general de las cooperativas multinacionales, emplazamos a la investigación futura a seguir proporcionando estudios detallados sobre la difusión transnacional de prácticas organizacionales en cooperativas que operen en otros países e industrias, teniendo en cuenta además las perspectivas de los diferentes actores que conforman estas organizaciones.

\section{Referencias bibliográficas}

Aguzzoli, R., y Geary, J. (2014) An 'emerging challenge': The employment practices of a Brazilian multinational company in Canada. Human Relations, $\mathrm{N}^{\circ}$ 67, pp. 587-609.

Andersson, U. y Holm, U. (2010) Managing the contemporary multinational. Cheltenham, UK: Edward Elgar.

Andrade, A.D. (2009) Interpretive Research Aiming at Theory Building: Adopting and Adapting the Case Study Design. The Qualitative Report, No 14, Vol. 1, pp. 42-60.

Arzadun, P. (2015) Globalización del proostebceso productivo establecido y su impacto socioeconómico: el caso de Orkli S. Coop. en China. Ciriec-España, Revista de Economía Pública, Social y Cooperativa, No 83, pp. 169-199.

Atzeni, M. y Ghigliani, P. (2007) Labour process and decision-making in factories under workers' self-management: empirical evidence from Argentina. Work, Employment and Society, № 21, Vol. 4, pp. 653-71.

Azkarraga, J., Cheney, G. y Udaondo, A. (2012) Workers Participation in a Globalized Market: Reflections on and from Mondragon. En: ATZENI, M. Alternative Work Organisations. New York: Palgrave Macmillan, pp. 76-102.

Bakaikoa, B., Errasti, A. y Begiristain, A. (2004) Gobierno y Democracia en los Grupos Empresariales Cooperativos ante la Globalización. El caso de Mondragón Corporación Cooperativa. Ciriec-España, Revista de Economía Pública, Social y Cooperativa, N ${ }^{\circ}$ 48, pp. 53-77. 
Bakaikoa, B., Etxezarreta, E. y Morandeira, J. (2013) Regeneración de Empresas de la Economía Social: Un reto en el País Vasco. REVESCO. Revista de Estudios Cooperativos, No 112, pp. 151-175. DOI: 10.5209/rev_REVE.2013.v112.43064.

Baxter, P. y Jack, S. (2008) Qualitative case study methodology: Study design and implementation for novice researchers. The Qualitative Report, $\mathrm{N}^{\mathrm{o}} 13$, Vol. 4, pp. 544 559.

Bretos, I. y Errasti, A. (2016) Dinámicas de regeneración en las cooperativas multinacionales de Mondragón: la reproducción del modelo cooperativo en las filiales capitalistas. Ciriec-España, Revista de Economía Pública, Social y Cooperativa, № 86, pp. 5-34.

Bretos, I. y Errasti, A. (2017) Challenges and Opportunities for the Regeneration of Multinational Worker Cooperatives: Lessons from the Mondragon Corporation-A Case Study of the Fagor Ederlan Group. Organization, No 24, Vol. 2, pp. 154-173.

Bretos, I. y Marcuello, C. (2017) Revisiting Globalization Challenges and Opportunities in the Development of Cooperatives, Annals of Public and Cooperative Economics, $\mathrm{N}^{\mathrm{o}} 88$, Vol. 1, pp. 47-73.

Cathcart, A. (2013) Directing democracy: Competing interests and contested terrain in the John Lewis Partnership. Journal of Industrial Relations, No 55, pp. 601-620.

Cheney, G. (2002) Values at Work. Ithaca: Cornell University Press.

Cheney, G., Cruz, I., Peredo, A.M. y Nazareno, E. (2014) Worker cooperatives as an organizational alternative: Challenges, achievements and promise in business governance and ownership. Organization, No 21, pp. 591-603.

Clamp, C. (2000) The Internationalization of Mondragon. Annals of Public and Cooperative Economics, $\mathrm{N}^{\mathrm{o}} 71$, pp. 557-577.

Clark, K., y Lengnick-Hall, M. L. (2012) MNC practice transfer: Institutional theory, strategic opportunities and subsidiary HR configuration. International Journal of Human Resource Management, $\mathrm{N}^{\mathrm{o}}$ 23, Vol. 18, pp. 3813-3837.

Dörrenbächer, C. y Geppert, M. (2011) Politics and power in the multinational corporation. Cambridge: Cambridge University Press

Eisenhardt, K.M. y Graebner, M.E. (2007) Theory building from cases: opportunities and challenges. Academy of Management Journal, No 50, pp. 25-32.

Elortza, N., Alzola, I. y Lopez, U. (2012) La gestión de la crisis en la Corporación Mondragón. Ekonomiaz, No 79, pp. 59-82.

Errasti, A. (2013) Tensiones y oportunidades en las multinacionales coopitalistas de Mondragón: el caso Fagor Electrodomésticos, Sdad. Coop. REVESCO. Revista de Estudios Cooperativos, No 113, pp. 30-60. DOI: 10.5209/rev_REVE.2014.v113.43385.

Errasti, A. (2015) Mondragon's Chinese Subsidiaries: Coopitalist Multinationals in Practice. Economic and Industrial Democracy, No 36, pp. 479-499.

Errasti, A., Bretos, I. y Etxezarreta, E. (2016) What do Mondragon Coopitalist Multinationals Look Like? The Rise and Fall of Fagor Electrodomésticos S. Coop. and its European Subsidiaries. Annals of Public and Cooperative Economics, No 87, pp. 433-456.

Errasti, A., Bretos, I. y Nunez, A. (2017) The Viability of Cooperatives: The Fall of the Mondragon Cooperative Fagor. Review of Radical Political Economics, No 49, Vol. 2, pp. 181-197.

Errasti, A., Heras, I., Bakaikoa, B. y Elgoibar, P. (2003) The Internationalization of Cooperatives: the Case of the Mondragon Co-operative Corporation. Annals of Public and Co-operative Economics, No 74, pp. 553-584.

FAGOR EDERLAN (2009) Sustainability Report, 2008. Eskoriatza: Fagor Ederlan. 
FAGOR EDERLAN (2016) Annual Report, 2015. Eskoriatza: Fagor Ederlan.

Ferner, A., Almond, P. y Colling, T. (2005) Institutional theory and the cross-national transfer of employment policy: The case of 'workforce diversity' in US multinationals. Journal of International Business Studies, No 36, pp. 304-321.

Ferner, A., Edwards, T. y Tempel, A. (2012) Power, institutions and the cross-national transfer of employment practices in multinationals. Human Relations, $\mathrm{N}^{\mathrm{o}}$ 65, pp. 16387.

Flecha, R. y Ngai, P. (2014) The challenge for Mondragon: Searching for the co-operative values in times of internationalization. Organization, $\mathrm{N}^{\mathrm{o}} 21$, pp. 666-82.

Flecha, R. y Soler, M. (2014) Communicative Methodology: Successful Actions and Dialogic Democracy. Current Sociology, No 62, pp. 232-242.

Forcadell, F.J. (2005) Democracy, Cooperation and Business Success: The Case of Mondragón Corporación Cooperativa. Journal of Business Ethics, $\mathrm{N}^{\mathrm{o}}$ 56, pp. 255-274.

Gephart, R. (2004) Qualitative Research and the Academy of Management Journal. Academy of Management Journal, No 47, pp. 454-462.

Heras, I. (2014) The Ties that Bind? Exploring the Basic principles of Worker-Owned Organizations in Practice. Organization, No 21, pp. 645-665.

Holm, U., Malmberg, A. y Solvell, O. (2003) Subsidiary Impact on Host-Country Economies: the Case of Foreign-Owned Subsidiaries Attracting Investment into Sweden. Journal of Economic Geography, No 3, pp. 389-408.

Hsieh, H.F. y Shannon, S. (2005) Three approaches to qualitative content analysis. Qualitative Health Research, $\mathrm{N}^{\mathrm{0}}$ 15, pp. 1277-1288.

Kostova, T. (1999). Transnational transfer of strategic organizational practices: A contextual perspective. Academy of Management Review, № 24, pp. 308-24.

Kostova, T. y Roth, K. (2002) Adoption of an organizational practice by subsidiaries of multinational corporations: Institutional and relational effects. Academy of Management Journal, No 45, pp. 215-233.

Kostova, T., Roth, K. y Dacin, M.T. (2008) Institutional theory in the study of multinational corporations: A critique and new directions. Academy of Management Review, No 33, Vol. 4, pp. 994-1006.

Luzarraga, J.M. (2008) Mondragon Multi-Localisation Strategy: Innovating a Human Centred Globalisation. Tesis doctoral, Mondragon Unibertsitatea.

Luzarraga, J.M. y IRIZAR, I. (2012) La Estrategia de Multilocalización Internacional de la Corporación Mondragón. Ekonomiaz, No 79, pp. 114-145.

Luzarraga, J., Aranzadi, D. e Irizar, I. (2007) Understanding Mondragon globalization process: local job creation through multi-localization. 1st CIRIEC International Research Conference on the Social Economy. Victoria, Canadá, 22-25 de octubre de 2007.

Macleod, G. y Reed, D. (2009) Mondragon's Response to the Challenges of Globalization: a Multi-localization Strategy. En: REED, D. y MCMURTRY, J. Co-operatives in a Global Economy. Newcastle: Cambridge Scholars Publishing.

Mcmurtry, J. y Reed, D. (2009) Co-operatives in a Global Economy. Newcastle: Cambridge Scholars Publishing.

MONDRAGÓN (2016) Annual Report 2015. Eskoriatza: Corporación Mondragón.

Morrison, E.W. y Milliken, F.J. (2000) Organizational Silence: A Barrier to Change and Development in a Pluralistic World. Academy of Management Review, No 25, pp. 706725.

Narvaiza, L., Aragón Amonarriz, C., Iturrioz Landart, C., Bayle Cordier, J., y Stervinou, S. (2017) Cooperative Dynamics during the Financial Crisis: Evidence From Basque and 
Breton Case Studies. Nonprofit and Voluntary Sector Quarterly, № 46, Vol. 3, pp. 505524.

Novkovic S. y Sena V. (2007) Co-operative firms in global markets. Oxford: JAI.

Paranque, B. y Willmott, H. (2014) Cooperatives — saviours or gravediggers of capitalism? Critical performativity and the John Lewis Partnership. Organization, No 21, pp. 604625.

Parker, M., Cheney, G., Fournier, V. y Land, C. (2014) The Routledge Companion to Alternative Organization. London: Routledge.

Perlmutter, H. (1969) The tortuous evolution of the multinational enterprise. Columbia Journal of World Business, № 4, pp. 9-18.

Quintanilla, J., Susaeta, L., y Sánchez-Mangas, R. (2008) The Diffusion of Employment Practices in Multinationals: 'Americaness' within US MNCs in Spain? Journal of Industrial Relations, № 50, pp. 680-696.

Radrigán, M. y Barría, C. (2007) El rol de las cooperativas en un mundo globalizado. Quebec: Irecus.

Rosenzweig, P.M. y Nohria, N. (1994) Influences on human resource management practices in multinational corporations. Journal of International Business Studies, $\mathrm{N}^{\mathrm{o}}$ 25, pp. 229-51.

Sarria-Icaza, A.M. (2008) Economía solidaria, acción colectiva y espacio público en el sur de Brasil. Louvain-la-Neuve: Presses Universitaires de Louvain.

Scott, W.R. (1995) Institutions and organizations. Thousand Oaks: Sage.

Siebel, S. (2016). Cooperative economies in a global age. Tesis doctoral, RMIT University, Melbourne, Australia.

Spear, R. (2004) Governance in democratic member-based organizations. Annals of Public and Cooperative Economics, $\mathrm{N}^{\mathrm{o}} 75$, pp. 33-60.

Stake, R.E. (2005) Qualitative case studies. En: Denzin, N.K. y Lincoln, Y.S. (Eds.) The Sage Handbook of Qualitative Research, pp. 443-466. Thousand Oaks: Sage.

Taylor, S. (2007) Creating social capital in MNCs: The international human resource management challenge. Human Resource Management Journal, No 17, pp. 336-54.

Varman, R. y Chakrabarti, M. (2004) Contradictions of Democracy in a Workers' Cooperative. Organization Studies, No 25, Vol. 2, pp. 183-208.

Villarreal-Larrinaga, O. (2017) Is it desirable, necessary and possible to perform research using case studies? Cuadernos de Gestión, No 17, Vol. 1, pp. 147-172.

Welch, C., Piekkari, R., Plakoyiannaki, E., y Paavilainen-Mäntymäki, E. (2011) Theorising from case studies: Towards a pluralist future for international business research. Journal of International Business Studies, No 42, Vol. 5, pp. 740-762.

Xiaowen, T. (2007) Managing International Business in China. Cambridge: Cambridge University Press.

Yin, R.K. (2013) Case Study Research: Design and Methods. London: Sage Publications. 